\title{
Detection of human tumor cells by amplicon fusion site polymerase chain reaction (AFS-PCR)
}

\author{
Axel Weber, Sylvia Taube, Sven Starke, Eckhard Bergmann, \\ Nina Merete Christiansen, and Holger Christiansen
}

Department of Pediatric Oncology, Hematology, and Hemostaseology, Children's Hospital, University of Leipzig, Leipzig, Germany.

\begin{abstract}
Reliable diagnostic strategies for individuals with cancer demand practical methods for highly sensitive and specific detection of tumor cells. Amplification of genomic regions that include putative oncogenes is common in tumor cells of various types. Genomic array platforms offer the opportunity to identify and precisely map amplified genomic regions (ampGRs). The stable existence of these tumor cell-specific genomic aberrations during and after therapy, in theory, make ampGRs optimal targets for cancer diagnostics. In this study, we mapped ampGRs around the proto-oncogene MYCN of human neuroblastomas using a high-resolution tiling array (HR-TA). Based on the HR-TA data, we were able to precisely describe the telomeric and centromeric borders of the ampGRs and deduce virtual fusion sites of the joined ampGRs (amplicon fusion sites [AFSs]). These AFSs served as blueprints for the subsequent design of AFS bridging PCR assays (AFS-PCRs). Strikingly, these assays were absolutely tumor cell specific and capable of detecting 1 tumor cell in $1 \times 10^{6}$ to $8 \times 10^{6}$ control cells. We successfully proved the in vivo practicability of AFS-PCR by detecting and quantifying the specific AFS DNA of human MYCN-amplified neuroblastomas in the patients' corresponding peripheral blood and bone marrow samples. Thus, we believe AFS-PCR could become a powerful and nevertheless feasible personalized diagnostic tool applicable to a large number of cancer patients, including children with $M Y C N$-amplified neuroblastomas.
\end{abstract}

\section{Introduction}

The detection of minimal amounts of tumor cells within putative metastatic sites - LNs, BM, peripheral blood (PB), and cerebral fluid - is becoming indispensable for thorough stage classification and therapy stratification for all different kinds of malignancies. Most evidence that a very small residual population of tumor cells during or after therapy can significantly influence the final outcome of a patient is given from hematological malignancies. The early detection of such minimal amounts of residual or recurrent malignoma cells has been shown to be a major prognostic factor for adult and childhood acute lymphoblastic leukemia $(1,2)$. Among solid tumors, the clinical relevance of minimal residual disease (MRD) during and after therapy has been most extensively studied in breast cancer patients $(3,4)$. Smaller studies report a significant impact on prognosis for minimal numbers of tumor cells or tumor cell DNA detected in the BM or PB, respectively, for different types of nonhematological malignancies $(3,5,6)$.

Besides their use in clinical diagnostics, another important application for highly sensitive methods of tumor cell tracking is the screening of autologous stem cell or BM grafts for residual, potentially viable tumor cells to avoid retransfusion following myeloablative therapy (7). However, the current literature lacks feasible diagnostic strategies for most solid tumor entities with tumor cell specificity and sensitivity comparable to that commonly described for leukemia diagnostics.

Absolute tumor cell specificity is a characteristic of particular parts of the sequence of genomic amplifications, in which single copies of amplified genomic regions (ampGRs) directly attach,

Conflict of interest: The authors have declared that no conflict of interest exists. Citation for this article: J Clin Invest. 2011;121(2):545-553. doi:10.1172/JCI44415. forming amplicon fusion sites (AFSs). A major advantage of identifying genomic amplifications compared with other genetic aberrations is the possibility of an easy-to-perform screen for ampGRs via whole-genome comparative genomic hybridization (CGH) analysis. ampGRs are easily detectable in primary tumor specimens, even in those containing a high content of stromal tissue. Moreover, ampGRs are found in a wide variety of tumor entities, emphasizing the relevance of a diagnostic tool targeting these numerical aberrations (8). Recent integrated genomic analyses of breast cancer, colorectal cancer, and glioblastoma multiforme describe detectable ampGRs in almost every individual malignoma $(9,10)$. At least 1 ampGR (>12 copies per haploid genome) was found in 41 of 45 (91\%) breast cancer, 27 of 36 (75\%) colon cancer, and 18 of 22 (82\%) glioblastoma specimens. The described attributes suggest genomic amplifications may be a predetermined target for cancer diagnostics.

Since its discovery in 1983 (11), amplification of the MYCN locus on chromosome 2p24-25 in human neuroblastomas has emerged as one of the most intensively studied examples of gene amplification. We used human neuroblastoma cell lines and primary neuroblastomas harboring chromosome 2p24-25 amplifications as a model to describe a diagnostic algorithm to detect and map the ampGRs, and to use them as blueprints for the subsequent design of an AFS bridging, and thus absolute tumor cell specific, PCR assay (AFS-PCR; Figure 1).

\section{Results}

High-resolution tiling array (HR-TA) mapping of ampGRs. Based on HR-TA analysis of the DNA of 3 MYCN-amplified neuroblastoma cell lines and 40 primary neuroblastomas, we could describe 2 main types of amplicons (Figure 2 and Supplemental Figures 1 


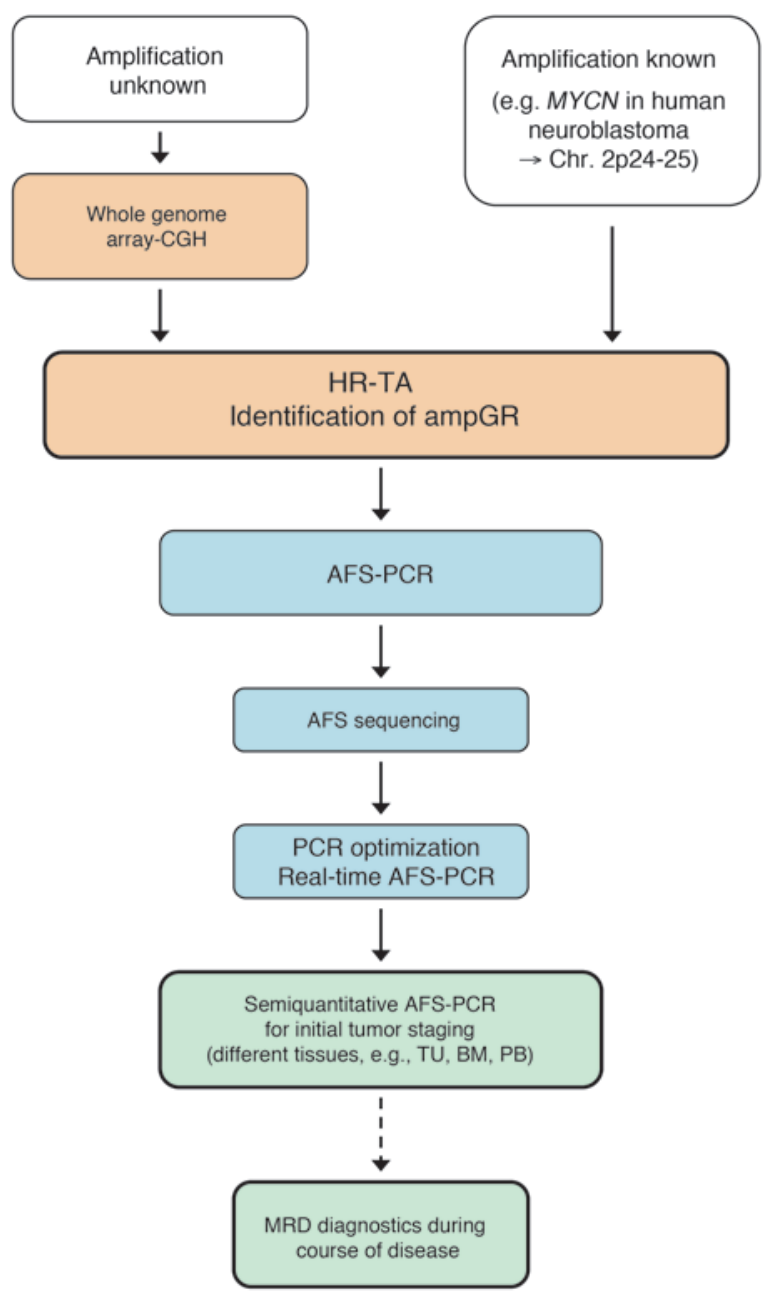

and 2; supplemental material available online with this article; doi:10.1172/JCI44415DS1). Of the 43 investigated MYCN amplicons, 27 consisted of 1 single ampGR (type 1 amplicons; Supplemental Figure 1), whereas the remaining 16 were assembled of 2 or more ampGRs (type 2 amplicons) that appeared to be separated by nonamplified genomic regions, potentially reflecting a more complex amplicon formation (Supplemental Figure 2).

Telomeric and centromeric borders of the identified ampGRs were unique for each tumor and cell line investigated. The ampGRs including the MYCN gene were defined as core ampGRs. The additional ampGRs of the type 2 amplicons were defined as satellite ampGRs. The telomeric borders of the core ampGRs extended from bp $10,446,277$ to bp $15,994,874$ (median, bp $15,717,248$ ). The centromeric borders extended from bp 16,041,166 to bp 21,821,063 (median, bp 16,474,239). We found the MEIS1 gene amplified within 1 ampGR of the IMR-32 cell line, as previously described (12-14). Just one other MYCN amplicon (tumor no. TU-26) showed integration of an ampGR in relative proximity to, but not including, the MEIS1 gene (bp 63,352,423 to bp 63,921,178; Supplemental Table 1). The intensities of the array signal of the ampGRs correlated with the copy numbers of the ampGRs estimated by comparative PCR, as described in our previous publication (15), with a Pearson correlation of 0.70 (Supplemental Table 2). The unique dimensions of the ampGRs permitted the design of

\section{Figure 1}

Identification and diagnostic use of AFSs in newly diagnosed malignomas. The diagnostic algorithm combines the possibility of screening for ampGRs by whole-genome CGH analysis, the precision of HR-TA as a platform for subsequent base-exact AFS mapping, the absolute specificity of the AFSs for the respective malignoma cells, and the high sensitivity of real-time PCR. For malignomas with already described ampGRs, isolated genomic DNA can be hybridized directly on the corresponding HR-TA, covering the region of interest. If no ampGRs are described, or common ampGRs are not found in preliminary investigations, whole-genome CGH analysis could be used to screen for regions with ampGRs for further fine mapping on a HR-TA. Once the borders of the ampGRs are mapped, AFS-PCR, and subsequently AFS-RQ-PCR, can be designed. AFS-PCR can be used as a feasible tool in the initial staging of malignoma diseases and, furthermore, for highly sensitive and absolutely specific tracking of remaining or recurrent malignoma cells during the course of the diseases.

individual PCR assays for the corresponding tumor cells, and thus for each investigated patient or cell line.

Creation of virtual AFSs and first primer design. Based on the exact information about the chromosomal location of the oligonucleotides that were printed on the HR-TA slides, we were able to assess virtual centromeric and telomeric borders of the ampGRs for each investigated neuroblastoma DNA. The virtual ampGR borders were predicted with a mean accuracy of 65 bp that was given by the HR-TA resolution. Based on the hypothesis that the single amplicons consist of consecutively joined copies of ampGRs, we were able to estimate possible resulting AFSs. Figure 3 shows an example of type 1 amplicons, with the KELLY cell line. In the first step, the AFSs were virtually simulated by joining the first 1,000 amplified bp of the virtual telomeric border to the last 1,000 amplified bp of the virtual centromeric ampGR border (Figure $3 \mathrm{~B})$. The sequences of these virtual junctions served as templates for the AFS-PCR primer design. A first generation of PCR primers was designed by setting one primer within the centromeric border region and the other within the telomeric border region of the ampGR. Thus, a successful AFS-PCR was only possible with the AFS bridging sequence present in the input DNA (Figure 3C). These AFSs were defined as type 1 AFSs. Interestingly, in all identified type 1 AFSs, the ampGRs were found to be joined in the headto-tail orientation (Supplemental Figure 3A).

Type 2 amplicons offered additional opportunities to design specific AFS-PCRs. Joining the virtual borders of 2 different ampGRs led to different possible virtual AFSs that could be used to design PCR primers analogously to type 1 AFSs. An example is given with the IMR-32 cell line (Figure 4). In type 2 AFSs, the ampGRs were found to be arranged in head-to-tail, head-to-head, or tail-to-tail orientation (Supplemental Figure 3B). Thus, in some cases, we found 2 different ampGRs to be joined inversely in their orientation. This resulted in a joining of the coding and noncoding DNA strands of 2 different ampGRs in opposite directions. Interestingly, additional short sequences were found between ampGRs in the 2 identified AFSs of IMR-32 and in different primary neuroblastomas, most of which were too short to identify their chromosomal origin with any precision (Figure 4C). Not all possible AFSs could have been successfully verified by PCR for the IMR-32 cell line and for primary neuroblastomas with complex type 2 amplicons (Supplemental Table 1).

Finally, the generation of at least 1 AFS-PCR leading to a specific PCR product was successful in 34 of 40 (85\%) primary neuroblas- 


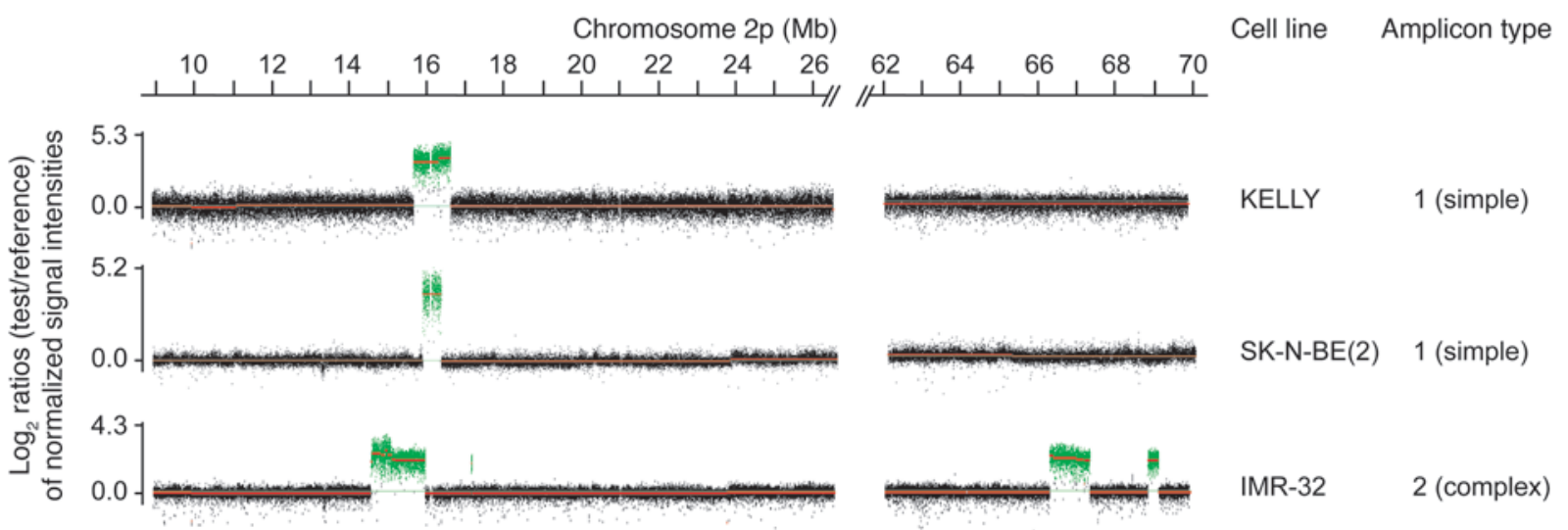

\section{Figure 2}

ampGRs of the $3 \mathrm{MYCN}$-amplified neuroblastoma cell lines around the MYCN gene on human chromosome 2. Signal-Map software (see Methods) was used for visualization of the HR-TA data. The relative copy number for each printed oligonucleotide is presented as fluorescence intensity of the Cy5-labeled test DNA (cell lines) normalized to the Cy3-labeled reference DNA (healthy human female) (log 2 ratios). The mean signal intensity values of the continuous genomic regions calculated are indicated by red lines. The 0 baseline is indicated by the green line. AmpGRs are highlighted green. Each cell line harbored an individual amplicon composed of different copy numbers of 1 (type 1) or more (type 2) ampGRs with unique telomeric and centromeric borders. See Supplemental Figures 1 and 2 for ampGRs of the $40 \mathrm{MYCN}$-amplified primary neuroblastomas.

tomas. Including AFS-PCR for the $3 \mathrm{MYCN}$-amplified cell lines, overall success was 37 of 43 (86\%) MYCN amplicons investigated. The design of AFS-PCR was more successful in type 1 amplicons (26 of 27) compared with type 2 amplicons (11 of 16). A detailed description of the single amplicons is given in Supplemental Figures 1 and 2 and Supplemental Tables 1 and 2 .

AFS sequencing. By sequencing the AFS-PCR products, we were able to exactly describe individual AFSs on the level of sequence. The coordinates of the ampGR borders of all successfully sequenced AFSs are available in Supplemental Table 1. A comparison of the sequenced AFSs with the AFSs predicted by the array CGH revealed a mean difference of $56 \mathrm{bp}$, which nearly matched the average array resolution of $65 \mathrm{bp}$ between the printed oligonucleotides. The maximum difference found was $354 \mathrm{bp}$.

Based on the exact sequences we were able to design primers located closer to the AFSs for real-time quantitative PCR (RQ-PCR).

Specificity of AFS-RQ-PCR. Based on the uniqueness of the individual ampGRs, each AFS-PCR product was absolutely specific for the neuroblastoma cell line or tumor on whose virtual AFS sequence it was designed. For the $3 \mathrm{MYCN}$-amplified cell lines, the specificity of AFS-PCR products is shown in Figure 5. Every AFS-PCR primer pair for the amplicons of the primary neuroblastomas were tested with control DNA isolated from human placental tissue. Strikingly, specific AFS-PCR product could only be identified with the corresponding tumor DNA (Supplemental Figure 3).

Sensitivity of AFS-RQ-PCR. The sensitivity of AFS-RQ-PCR was tested with dilution series of 3 human neuroblastoma cell lines with MYCN amplification [IMR-32, KELLY, and SKnBE(2)] with a line without MYCN amplification (SH-EP; see Methods for details). These MYCN-amplified cell lines and SH-EP cells harbor almost identical DNA content per cell. The $\Delta \mathrm{Ct}$ values of the specific AFSPCR products relative to the control PCR product, a fragment of inhibin- $\beta$-b $(I N H B B)$, were additionally set in relation to the DNA of the nondiluted MYCN-amplified cell lines, calculated according to the $2^{-\Delta \Delta \mathrm{Ct}}$ method (see Methods and ref. 16). Strikingly, the calculated values of the successive dilution steps reflected the single dilution steps of the input DNA (Figure 6 and Supplemental Fig- ures 4 and 5). For all 3 cell lines, AFS-PCR products were reliably detectable in the last dilution step: $1 M Y C N$-amplified cell per $10^{6}$ $\mathrm{SH}-\mathrm{EP}$ cells. We further escalated testing for sensitivity with a successive dilution of DNA of the $1 \times 10^{-6}$ dilution steps with SH-EP control DNA at a ratio of 1:1. Finally, the method provided suitable to track the AFS from $1 \mathrm{MYCN}$-amplified cell of $8 \times 10^{6}$ control cells. The control for a specific AFS-PCR product, as assessed by agarose gel electrophoresis, was positive down to the highest dilution step (Supplemental Figure 4). Thus, we estimated the sensitivity for AFS-PCR to detect $1 \mathrm{MYCN}$-amplified cell of $1 \times 10^{6}$ to $8 \times 10^{6}$ control cells within our experimental setting.

Practicability for MRD diagnostics in primary neuroblastomas. For 3 of the 40 primary neuroblastomas investigated, we were able to investigate DNA from tissue of a second biopsy or of recurrent disease by HR-TA (Supplemental Figure 7). In all 3 cases, we found absolute consistent borders of the ampGRs in the primary tumor tissues and the tissue of each secondary specimen, evidence supporting the structural stability of the ampGRs over time.

To test the practicability of AFS-PCR to identify tumor cells or tumor cell DNA in tissues other than primary tumor tissue, we investigated genomic DNA of BM, PB, and residual tumor tissue during the treatment of 3 different neuroblastoma patients. Strikingly, we were able to detect AFS DNA in different sample types from all 3 neuroblastoma patients investigated (Figure 7 and Supplemental Figure 6). From patient TU-23, we were able to investigate BM samples from the time of the initial diagnosis as well as from BM aspirations after the first cycle of chemotherapy. The relative decrease of the number of tumor cells detected in the secondary BM samples of this patient (Figure 7) might indicate a therapeutic effect. Because of the lack of exact information, the content of tumor cells in the initial tumor samples of patients TU-20, TU-21, and TU-23 was assumed to be $100 \%$ analogous to that of the described cell culture experiments. By general agreement, this does not reflect the situation in primary tumor specimens. The tumor cell content in the initial tumor sample is a variable attribute and depends on malignoma type and histological grading. Because for AFS-PCR, the number of tumor cells or tumor cell 
A

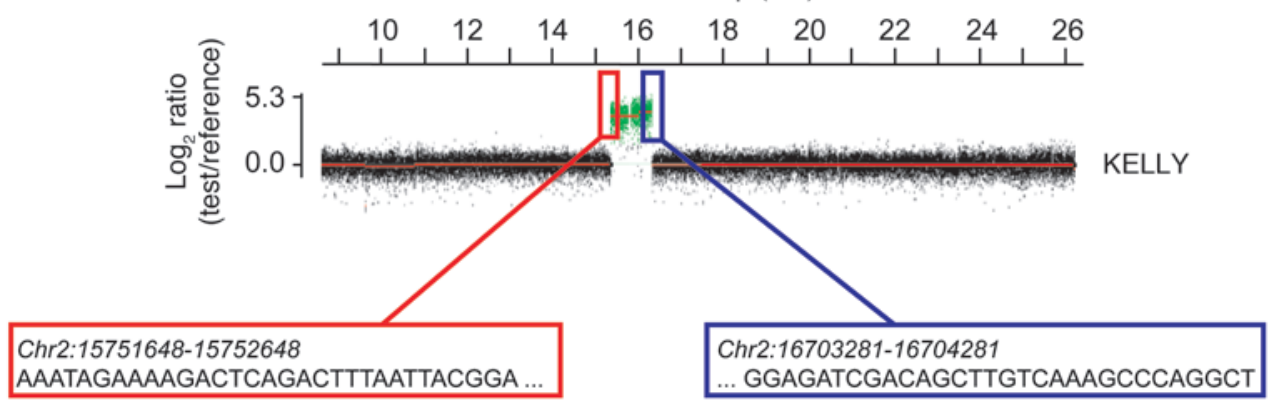

$\mathbf{B}$

GGAGATCGACAGCTTGTCAAAGCCCAGGCT

$?$

- AAATAGAAAAGACTCAGACTTTAATTACGGA ...

C
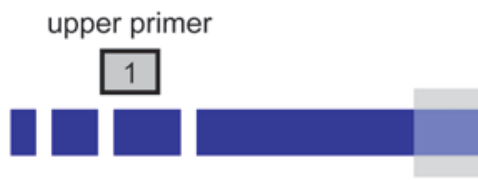

?

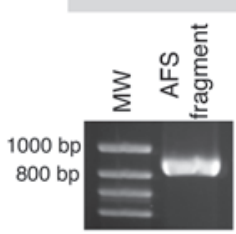

D

\begin{tabular}{|r|l|}
\hline Chr2: 16704345 & Chr2: 15751592 \\
... CCGGGTCTTCTTGGACTCTCCACTTGCTCTTGC & TCTTTACTTTCTGCTCAGTTTTGCTGTGAACCTA ... \\
\hline
\end{tabular}

E

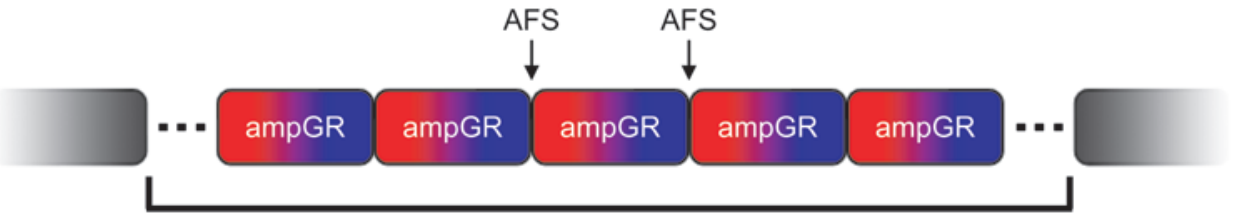

Amplicon

\section{Figure 3}

Identification of the AFS sequence in the type 1 amplicon of the KELLY cell line. (A) HR-TA data indicate the virtual telomeric and centromeric borders of the ampGR of the KELLY cell line by referring to the first and last amplified oligonucleotide. The relative copy number for each printed oligonucleotide is presented as fluorescent intensity of the Cy5-labeled test DNA (cell lines) normalized to the Cy3-labeled reference DNA (healthy human female) ( $\log _{2}$ ratios). The mean signal intensity values of the continuous genomic regions calculated are indicated by red lines. The ampGR is highlighted green. (B) The last (red) and first (blue) 1,000 amplified bps are fused together, resulting in a virtual AFS. (C) Primers for a first AFS-PCR are designed upand downstream of the virtual AFS. (D) Sequencing of the excised AFS fragment from both sides, using the previously designed primers, results in the exact AFS. (E) Simplified model of the architecture of a simple type 1 amplicon. ampGRs are subsequently joined together in head-to-tail orientation, resulting in a tumor cell-specific AFS. genomes relative to nonmalignant cell genomes in a given sample (e.g., $\mathrm{BM}$ and $\mathrm{PB}$ ) is calculated directly in relation to the $\Delta \mathrm{Ct}$ values of the primary tumor sample, it must be corrected for the tumor cell content of the primary tumor. This correction (see Methods for details) is needed in order to obtain interindividually comparable results. For example, this calculation is of importance to define threshold values of total cell numbers in a given compartment (e.g., BM) that might be of potential clinical relevance, like for a decision about changing a therapeutic strategy. Corrected results, taking into account different hypothetical tumor cell contents of the primary tumors investigated (Figure 7), are displayed in Supplemental Table 3.

\section{Discussion}

Once established from tissue of the primary tumor site, an individual AFS-PCR offers a powerful tool to track tumor cells or tumor cell DNA in potential metastatic sites of any tissue origin as well as in BM aspirates, in PB samples, and even in cerebral fluid, urine, or any other sample of the corresponding patient. Hence, AFS-
PCR could significantly improve the exact definition of the tumor stage at the time of initial diagnosis, the monitoring of response to therapy by quantification of tumor cells in subsequent samples, and the early detection of MRD or recurrent disease over time.

As fused ampGRs are a prerequisite for the development of an AFS-PCR, the diagnostic algorithm is limited to patients with malignancies harboring genomic amplifications (Figure 1). However, current publications give evidence that ampGRs could be found in a large percentage of different types of malignomas ( 9 , 10). A second prerequisite is the stability of the borders of the ampGRs. There are very few data about possible flexibility in the structure of ampGRs during the course of malignant diseases. Our own prior data from $M Y C N$-amplified neuroblastomas showed a stable coamplification pattern in almost all investigated subjects (15). However, the multiplex PCR used in this setting was characterized by low resolution and thus lacked satisfactory information on possible smaller changes in the amplicon structure. Nonetheless, it is unlikely that a complex architecture of consecutively repeated ampGRs might change its structure on the level of sequence dur- 
A

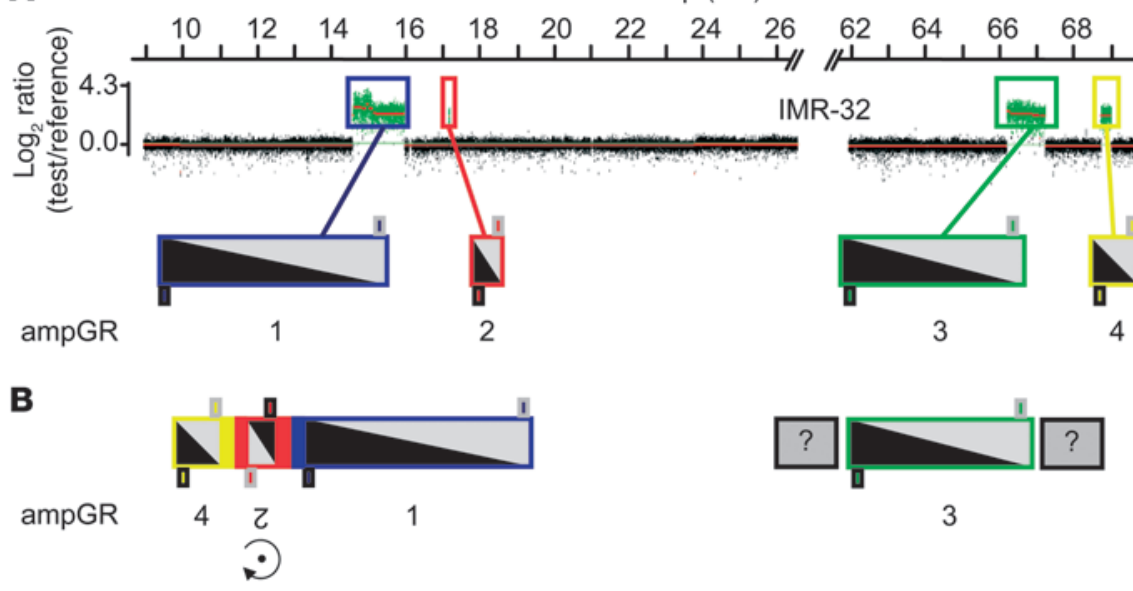

C

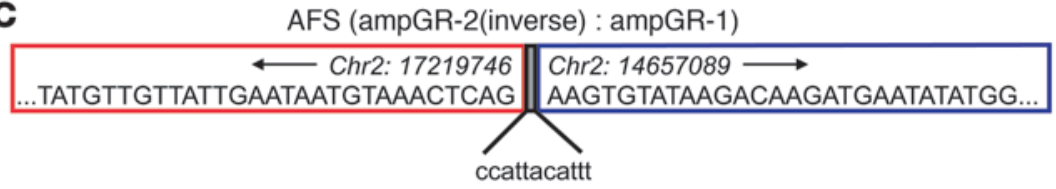

ccattacattt

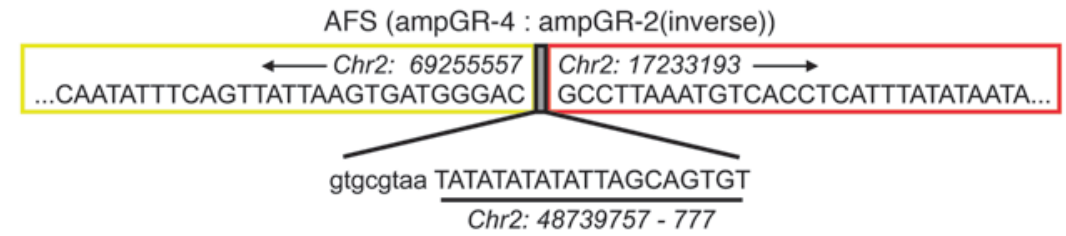

D

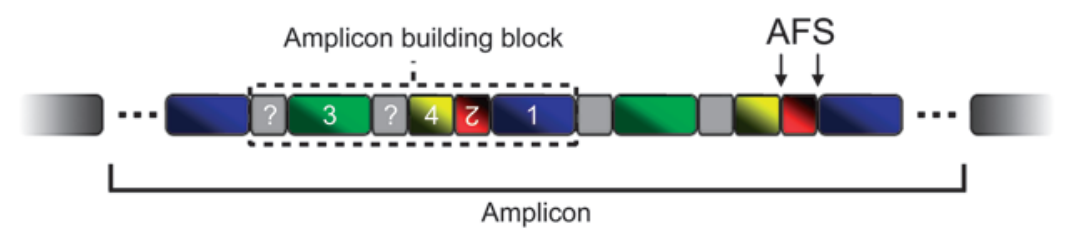

\section{Figure 4}

Identification of AFS sequences in the type 2 amplicon of the IMR-32 cell line. (A) HR-TA data indicate the virtual telomeric and centromeric borders of 4 ampGRs that appear to be separated by nonamplified genomic regions. (B) ampGR order within a single building block. ampGR-2 is fused between ampGR-1 and ampGR-4 inversely, as identified by different combinations of PCR primers and subsequent sequencing. No combination of primers for the known ampGRs successfully integrated ampGR-3 in the amplicon. Most likely, additional ampGRs that were not covered by the HR-TA are adjacent to both sides of ampGR-3, avoiding a successful PCR. (C) Sequencing of the AFS-PCR products from both sides resulted in the exact AFS. In both AFSs, additional short sequences were found between the ampGR borders. In the AFS between ampGR-4 and ampGR-2 (inverse), an additional ampGR, 20 bp long, was identified. For shorter sequences (lower case) of both AFSs, it was not possible to identify the definite chromosomal origin. (D) Simplified model of the architecture of a complex type 2 amplicon. Different ampGRs are joined together in head-to-tail, head-to-head, or tail-to-tail orientation, resulting in different tumor cell-specific AFSs. The different ampGRs build single building blocks that are subsequently joined together. The entirety of all ampGRs corresponds to the whole, individual amplicon. ing the course of disease and therapy, resulting in a change of all AFSs that were initially present.

Hypothetically, the monitoring of malignancies that obviously develop from more than 1 clonal cellular population could be at the limits of AFS-PCR. However, current publications indicate an initially monoclonal origin for different kinds of malignomas, even though a rising diversity of different subclones could be found during the course of disease $(17,18)$. Compared with other genomic aberrations, ampGRs showed high stability between different arising subclones in those studies. One of the neuroblastomas investigated (patient TU-19) harbored 2 different core ampGRs detected by HR-TA. There are 2 possible explanations for this finding: either the different ampGRs were existent in 1 single tumor cell clone, leading to a complex amplicon pattern with the different possible AFSs, or 2 independent cell clones were present in the initial tumor. Because it is impossible to easily discriminate between these 2 conditions, it is imperative to design PCR for the AFSs of all potential cell clones, since it might be possible that during the course of disease and therapy, one clone might become privileged, and thus only a single AFS-PCR could be of use for subsequent diagnostic steps. For tumor TU-19, we were able to successfully design AFS-PCRs for both possible amplicons, but because we lacked consecutive tissue probes of the corresponding patient, it was not possible to assess whether 1 of the 2 AFSs disappeared during the course of disease.

Different conditions may influence the ability to successfully establish an AFS-PCR for an individual malignancy. The most important prerequisite is DNA of good quality from the initial tumor site for HR-TA, as DNA quality directly influences the exact mapping of the ampGR borders. Depending on the resolution of the HR-TA, it is also possible to miss small additional ampGRs and thus fail to successfully design AFS bridging primers. In general, it is more difficult to identify the exact ampGR borders of tumors with just a few copies of ampGRs (gain) compared with high-copy amplifications. An example for a low-copy gain is given with tumor TU-4 (Supplemental Figure 1). Performing the HR-TA more than once in such cases could increase the reliability of the array-based prediction of the ampGR borders. Moreover, each AFS-PCR has to be optimized prior to its use for semiquantitative diagnostics based on the individual sequences flanking each AFS. For the complex type 2 amplicons, it is theoretically possible to design primers for all potential AFSs. For these cases, it is important to keep in mind that 2 different ampGRs could be merged in the same or the opposite direction with regard to the coding DNA strand (Supplemental Figure 3).

The used HR-TA with a custom design covering 8 megabases $(\mathrm{Mb})$ telomeric and $10 \mathrm{Mb}$ centromeric to $\mathrm{MYCN}$ (and additional 


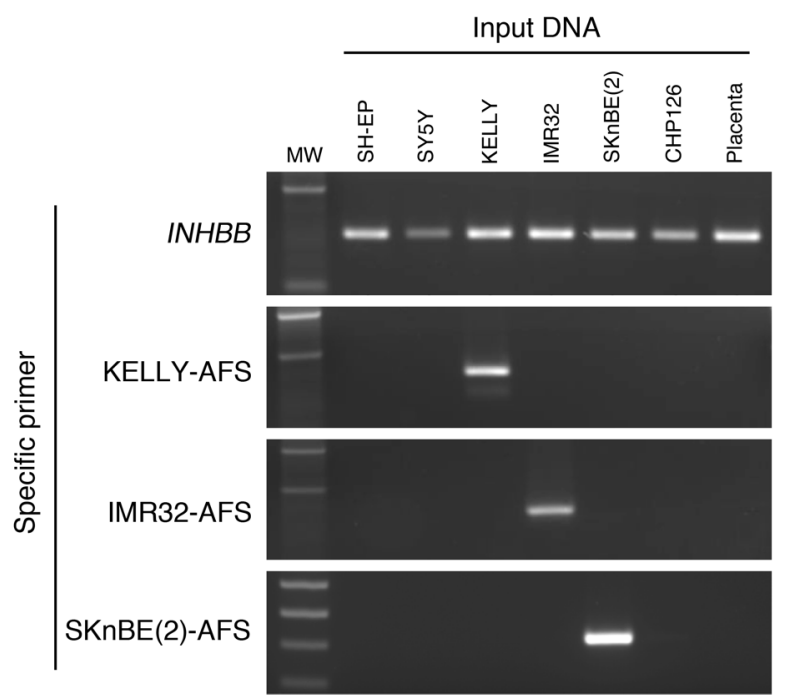

$10 \mathrm{Mb}$ around MEIS1) was suitable to design AFS bridging primers for at least $85 \%$ of all amplicons investigated. As shown for the MYCN amplicon of the IMR-32 cell line, ampGRs situated far from the core ampGRs were found integrated within an amplicon building block (ref. 14 and Figure 4). For some of the investigated neuroblastomas, 1 ampGR - or even more - might have been missed simply because the HR-TA did not cover the corresponding chromosomal regions. This would explain why it was impossible to design an AFS-PCR in these cases. Although successful in most cases in this study, the described identification of the AFSs was based on a simplified model of amplicon formation; thus, it is not possible to exclude the possibility that amplicons may be composed in a complex pattern that does not allow the investigator to successfully identify 1 single AFS based on the given HR-TA data (19).

If the described requirements are fulfilled, AFS-PCR offers remarkable benefits over applications currently used for the detection of minimal amounts of tumor cells (20). Compared with flow cytometry, which is mainly used for the detection of leukemic cells (21-23), AFS-PCR - like other PCR-based strategies - is characterized by higher sensitivity. However, even compared with different commonly used PCR applications, AFS-PCR is characterized by certain advantages. Thus, RQ-PCR strategies targeting the mRNA of genes that are primarily expressed in translines and further dilution steps.

\section{Figure 5}

Specificity of AFS-PCR. PCRs with genomic DNA of different neuroblastoma cell lines or human placenta tissue were performed with either specific AFS primers of 1 of the 3 investigated cell lines, or primers for a fragment of INHBB that served as control. $15 \mu \mathrm{l}$ of the PCR was separated in a $3 \%$ agarose gel by electrophoresis and visualized under UV light after ethidium bromide staining. AFS-PCR was absolutely specific for the respective cell line DNA harboring the individual ampGR. formed cells lack the reliable specificity that fundamentally has to be claimed for MRD diagnostics $(24,25)$. PCR assays designed to bridge the fusion sites of gene fusion products (e.g., BCR/ABL) are, like AFS-PCR, tumor cell specific $(22,23,26)$. However, in contrast to AFSs, the fusion sites of such gene fusion transcripts are not individual for each patient: cross-contamination between different patients' specimens is a known problem for these MRD
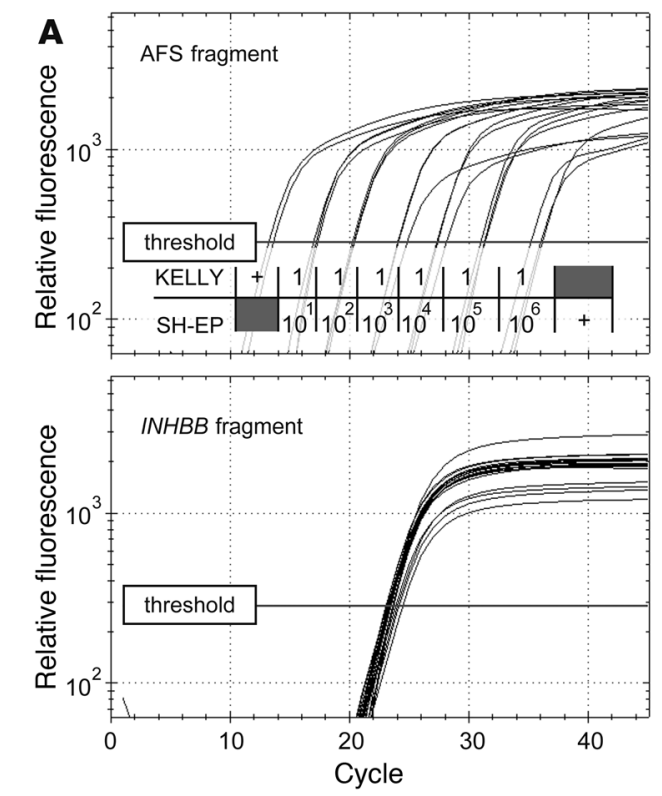

\section{Figure 6}

Sensitivity of AFS-PCR. (A) RQ-PCR traces of 1 representative dilution series of KELLY cells with SHEP cells. A $\log _{10}$ dilution series of suspended $M Y C N$-amplified cells (KELLY) with non-MYCN-amplified control cells (SH-EP) was performed in triplicate prior to DNA isolation. Undiluted KELLY cells (+) served as $100 \%$ tumor cell control; SH-EP cells (+) served as negative control. $200 \mathrm{ng}$ DNA isolated from $3 \times 10^{6}$ cells of each dilution step or control served as templates for RQ-PCR with either specific AFS primers or INHBB control primers. (B) Calculated $2^{-\Delta \Delta C t}$ of all 3 independent dilution series. Mean and SD are indicated by points and horizontal bars, respectively. The values reflected almost exactly the corresponding dilution steps of the KELLY cell line. See Supplemental Figures 4 and 5 for IMR-32 and SKnBE(2) cell 
A

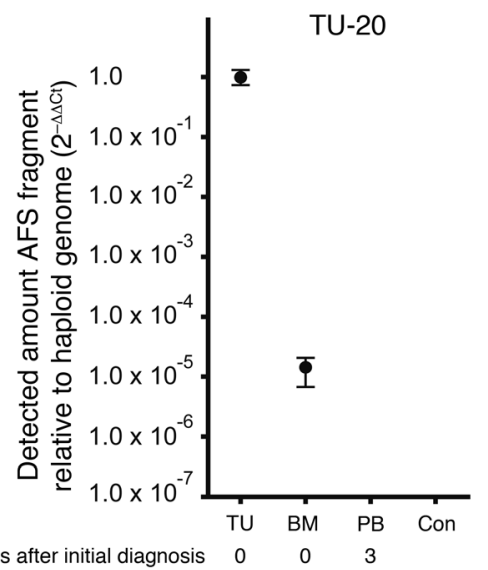

B

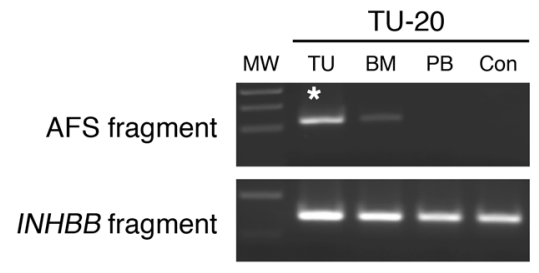

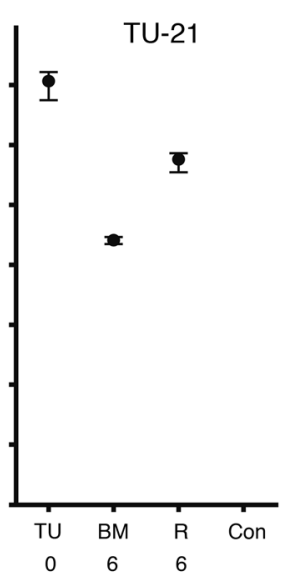
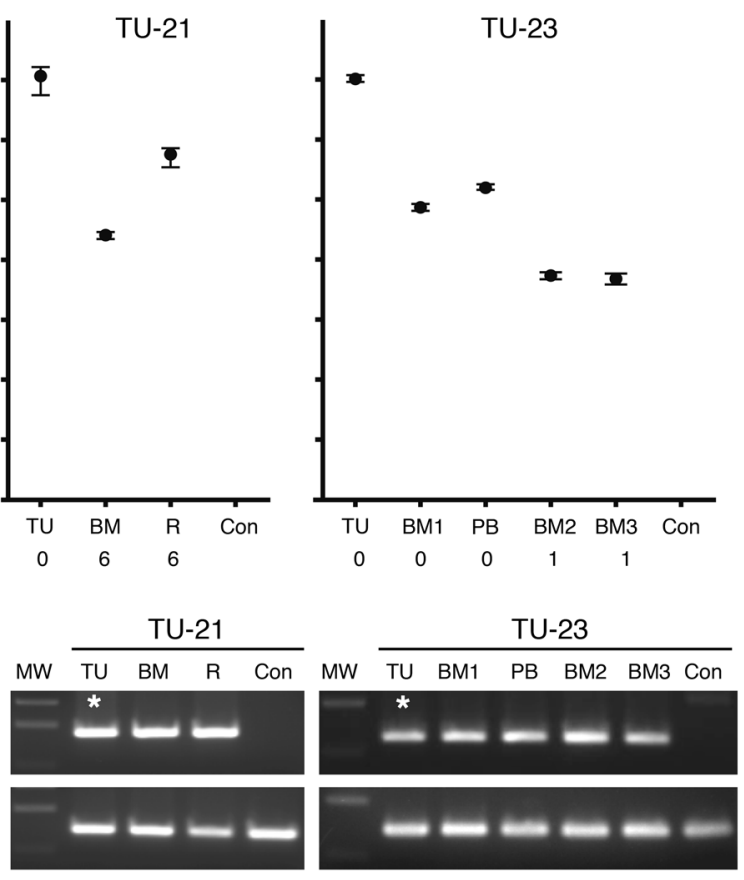

\section{Figure 7}

Specific AFS fragments are detectable in samples of different tissue origin of neuroblastoma patients at the time of initial diagnosis and during therapy. (A) Tumor cell contents of the primary tumors (TU) were estimated at $100 \%$. Tumor cell contents of the different probes were quantified according to the $2^{-\Delta \Delta C t}$ calculation. Each PCR was performed in triplicate. Mean and SD are indicated by points and horizontal bars, respectively. DNA from human placenta tissue served as negative control (Con). AFS-PCR identified a weak BM infiltration for patient TU-20 at the time of diagnosis. This infiltration was not detectable cytologically. Patient TU-21 had considerable BM infiltration in a control aspiration after 6 months of therapy. AFS-PCR of a secondary biopsy sample of the residual tumor $(\mathrm{R})$ showed a 10-fold decrease of tumor cells compared with the initial tumor specimen. Patient TU-23 had BM infiltration and circulating tumor cells in the PB at the time of diagnosis (BM1). The secondary BM aspirations, taken from 2 independent anatomical locations after 1 month of therapy (BM2 and BM3), showed a 10-fold decrease of tumor cells compared with the initial BM sample, giving evidence for a response to therapy. (B) AFS fragments and INHBB control fragments of the RQ-PCR separated in a $3 \%$ agarose gel. RQ-PCR had been running for 40 cycles. Asterisks denote lines of the tumor probe loaded with half the PCR product of the other lines.

targets. The dependency on mRNA as starting material for MRD diagnostics instead of genomic DNA is an additional disadvantage with regard to its stability and its isolation from tissue specimens. Furthermore, the identification of specific mRNA expression pattern, gene fusion products, or tumor cell-specific mutations, such as in putative tumor suppressor genes (27-29), may be hindered by a high content of stromal tissue in the specimen of interest, whereas the identification of ampGRs could still be possible. PCR strategies targeting rearrangements in genes encoding for immunoglobulin chains are of high specificity and sensitivity but, like flow cytometry, suffer from limitations in the detection of MRD in different kinds of leukemias (30).

Currently, the designs of genomic microarrays are becoming progressively higher in resolution, which results in several advantages. The gaps between the printed oligonucleotides on the available standard whole-genome arrays will be small enough for a direct AFS-PCR design, and customized tiling arrays for each single genomic region of interest will become dispensable. To reach this goal, arrays with $10^{7}$ or more printed oligonucleotides are required, whereas the recently available CGH arrays consist of a standard resolution of $2.1 \times 10^{6}$ printed probes resulting in gaps of 1.5-2.0 kilobases. Those arrays would be efficient in reflecting larger genomic regions around the core regions of interest, increas- ing the possibility of complete amplicon mapping even for type 2 amplicons. Decreasing costs of high-resolution array approaches will make this method more affordable for use in routine diagnostics. In the long term, next-generation sequencing has the potential to substitute for the array CGH. With this technique, it is still possible to directly sequence AFSs or other specific junction sites in the tumor cell genome, making them affordable for a direct PCR design $(31,32)$. However, this approach is so far too expensive and time intensive for use in routine diagnostics.

Fulfilling the qualitative requirements of different analytical methods, the malignoma cell-specific AFSs could also be useful for designing PCR primers or other types of probes targeting the AFSs directly (e.g., labeled probes for fluorescence in situ hybridization). Besides these diagnostic approaches, AFSs could also be of interest as a target for different malignoma cell-specific therapy strategies like double-strand DNA binding antisense oligonucleotides.

In summary, our approach gives rise to a feasible tool for absolutely specific and highly sensitive detection of tumor cells, suitable for a large number of patients suffering from different malignancies. Once established, it should be possible to easily perform AFS-PCR in every laboratory capable of performing RQ-PCR. The possibility to reliably detect ampGRs in a primary tumor specimen even with low tumor cell content by AFS- 
PCR represents a major advantage over other methods recently described. Its prognostic impact on MRD diagnostics and its role as a criterion for clinical decision making has to be evaluated for each single type of malignoma.

\section{Methods}

Patients. We studied primary tumor specimens from 40 children with $M Y C N$-amplified neuroblastomas diagnosed in Germany from 1986 to 2003. The 40 neuroblastomas were selected from a total of 230 tumor specimens with MYCN amplification within this time period based on the availability of tumor tissue of comparable quality for DNA isolation.

All neuroblastoma diagnoses were confirmed by histological assessment of a tumor specimen obtained from surgery. The tumors were classified according to INSS criteria (33). All patients were treated according to previously described protocols $(34,35)$, and informed consent for therapy and study procedures was obtained. The studies were reviewed and approved by the Ethics Committee of the medical faculty of the University of Cologne (Cologne, Germany).

Our study group consisted of 3 stage 1, 0 stage 2, 13 stage 3, 20 stage 4, and 4 stage $4 \mathrm{~S}$ tumors. The median patient age at diagnosis was 23.7 months (range, 0.03 to 78.6 months). The median follow-up time for all 40 patients was 31.9 months (range, 0.4 to 87.6 months). The median follow-up time of the patients that died from the disease was 11.5 months $(n=21)$, compared with 56.4 months for surviving patients $(n=19)$. Age, tumor stage, and survival data for each investigated patient are given in Supplemental Table 2 .

Cell culture. Human neuroblastoma cell lines SH-EP, IMR32, KELLY, and SKnBE(2) were grown under standard cell culture conditions in RPMI Medium (Gibco; Invitrogen) containing 10\% fetal calf serum (SigmaAldrich), glutamine $(2 \mathrm{mM})$, penicillin- $\mathrm{G}(100 \mathrm{U} / \mathrm{ml})$, and streptomycin $(100 \mu \mathrm{g} / \mathrm{ml})$ at $37^{\circ} \mathrm{C}$ and $5 \% \mathrm{CO}_{2}$. Cells were trypsinized and harvested at $80 \%$ confluence. For dilution series, the cells were counted, and MYCNamplified cells [IMR32, KELLY, and SKnBE(2)] were stepwise diluted with non-MYCN-amplified SH-EP cells according to the following dilution steps: $1 M Y C N$-amplified cell per 10 SH-EP cells (1:10), 1 MYCN-amplified cell per 100 SH-EP cells $\left(1: 10^{2}\right)$, followed by $1: 10^{3}, 1: 10^{4}, 1: 10^{5}$, and $1: 10^{6}$. For all $M Y C N$-amplified cell lines, the dilution series were performed in independent triplicates. $3 \times 10^{6}$ cells of each dilution step were then used for DNA isolation.

DNA isolation and further dilution steps. Isolation of genomic DNA was performed using the DNA-Blood and Tissue Kit (QIAGEN). DNA quality and concentration was measured spectrometrically (Nanodrop, Amersham).

To test the sensitivity, we further diluted the DNA of each $1: 10^{6}$ dilution step with SH-EP control DNA at a ratio of 1:1 down to $1: 80^{6}$ for each cell line investigated.

HR-TA design. Design of the printed oligos and manufacturing of the array slides was performed by Roche NimbleGen. 384,599 DNA oligos of 35-50 nucleotides in length were printed on 1 array slide. The genomic regions of interest were located on chromosome $2 \mathrm{p}$ (region 1 [around MYCN], bp $8,502,655$ to bp 26,522,939; region 2 [around MEIS1], bp 59,390,222 to bp $66,064,884$; region 3 [control], bp 120,730,406 to bp 120,948,861), covering $24,913,401 \mathrm{bp}$ in total. We obtained bp coordinates from the UCSC Genome Bioinformatics Database, March 2006 (NCBI36/hg18) assembly (http://genome.ucsc.edu/). The average resolution was 1 DNA oligo every $65 \mathrm{bp}$. The specific resolution for a genomic section was dependent on the underlying sequence. Thus, sections with long sequence repeats were represented in a lower resolution, while sections containing highly specific chromatin (e.g., gene encoding euchromatin) were represented in a higher resolution. Hybridization of the tiling array required a total of $5 \mu \mathrm{g}$ DNA (DNA quality criteria and hybridization methods were according to Ima-
Genes). The DNA of the neuroblastoma cell lines and primary tumors were hybridized together with control DNA (healthy human being) on the array slides. The DNA of the neuroblastoma specimens were labeled with Cy5, whereas the control DNA was labeled with Cy3. SignalMap Software (version 1.9; NimbleGen) was used for analysis of the HR-TA data.

Primer design. All primers for standard PCR and RQ-PCR were designed using OLIGO Primer analysis software (version 6.41; Molecular Biology Insights Inc.). See Results and the Figure 2 and Figure 3 legends for details of primer design. The sequences of all primers used are listed in Supplemental Table 4.

PCR and sequencing. The PCR for validation of the virtual AFSs was performed under standardized conditions. We used the QIAGEN HotStarTaq-Plus PCR Mastermix (12.5 $\mu \mathrm{l}), 200 \mathrm{ng}$ DNA, 2 primers at a final concentration of 1 pmol each, $1.5 \mu \mathrm{L}$ DMSO (Sigma-Aldrich), and Aquadest (Braun) at $25 \mu \mathrm{l}$. Cycler conditions were as follows: 5 minutes initial denaturation at $95^{\circ} \mathrm{C}$, followed by 38 cycles with 20 seconds at $95^{\circ} \mathrm{C}$, 20 seconds at $57^{\circ} \mathrm{C}$, and 40 seconds at $72^{\circ} \mathrm{C}$. Electrophoresis was performed in $1 \%-3 \%$ agarose gels dependent on the PCR fragment length. Gels were stained with ethidium bromide, and bands were visualized under UV light (Image Master VDS; Pharmacia).

Bands of estimated length were excised from the gel, and PCR fragments were isolated using the QIAGEN gel extracting kit following the manufacturer's instructions. Each AFS fragment was sequenced from both sides using a BigDye Terminator 3.1 Ready Reaction Cycle Seq Kit (Applied Biosystems) following the manufacturer's instructions. We used a 16 Capillary Sequenzer Genetic Analyzer 3100 from Applied Biosystems. $250 \mathrm{ng}$ of DNA and 10 pmol of 1 primer were put in 1 sequencing reaction.

RQ-PCR conditions were as follows: QIAGEN HotStarTaq-Plus PCR Mastermix (12.5 $\mu \mathrm{l}), 200 \mathrm{ng}$ DNA, 2 primers at a final concentration of 1 pmol each, $1.5 \mu$ l DMSO, $2.5 \mu \mathrm{l}$ SYBR-Green (10x concentration; Roche), and Aqua-dest at $25 \mu \mathrm{l}$. Cycler conditions were as follows: 5 minutes initial denaturation at $95^{\circ} \mathrm{C}$, followed by 40 cycles with 20 seconds at $95^{\circ} \mathrm{C}$, 20 seconds at $56^{\circ} \mathrm{C}-58^{\circ} \mathrm{C}$, and 40 seconds at $70^{\circ} \mathrm{C}-72^{\circ} \mathrm{C}$ (depending on individual primer binding conditions). All real-time PCR was performed on a BIORAD iQ5-Cycler. Each real-time PCR, including the internal control $I N H B B$, was performed in triplicate.

Statistics. Relative amounts of MYCN-amplified tumor cells within the different steps of the dilution series of the cell lines, or in the samples of different tissue origin from the neuroblastoma patients investigated, were calculated using the $2^{-\Delta \Delta \mathrm{Ct}}$ method (16). Therefore, the Ct values of the specific AFS fragments were normalized to the corresponding $\mathrm{Ct}$ values of the $I N H B B$ control PCR fragments. Each resulting $\triangle \mathrm{Ct}$ value was further normalized to the median $\triangle \mathrm{Ct}$ of the corresponding $100 \% M Y C N$-amplified cell line DNA or primary tumor DNA specimen. This calculation resulted in triplicate $2^{-\Delta \Delta C t}$ values for each specimen investigated. RQ-PCR data in Figure 7 present the mean and SD of these $2^{-\Delta \Delta C t}$ triplicates. Each dilution series of $M Y C N$-amplified cell lines was performed in independent biological triplicates. Thus, RQ-PCR data in Figure 6 and Supplemental Figure 4 present the mean and the $\mathrm{SD}$ of all 3 corresponding $2^{-\Delta \Delta \mathrm{Ct}}$ triplicates (i.e., 9 values).

To correct the Ct value of the specific AFS-PCR fragment of the primary tumor DNA to the histological assessed tumor cell content, we used the equation AFS-Ct $\mathrm{t}_{\text {corrected }}=\mathrm{AFS}-\mathrm{Ct}_{\text {native }}+\log _{2} X$; where $X$ is the relative tumor cell content of the primary tumor specimen (e.g., 0.8 for $80 \%$ ). All other Ct values stayed unchanged. $\Delta \mathrm{Ct}$ values were then calculated in relation to the corrected $\Delta \mathrm{Ct}$ value of the primary tumor specimen according to the $2^{-\Delta \Delta \mathrm{Ct}}$ method (Supplemental Table 3).

\section{Acknowledgments}

We thank our colleagues from about 100 German pediatric centers for providing us with neuroblastoma tumor material since 1981. 
We thank F. Berthold and B. Hero (University of Cologne, Cologne, Germany) for providing clinical data and F. Lampert (University of Giessen, Giessen, Germany) for supporting our research group. This work was substantially supported by the Deutsche Forschungsgemeinschaft (project no. WE-4324/1-1). We thank Steffi Meyer (University of Leipzig) for valuable comments on the manuscript.
Received for publication July 18, 2010, and accepted in revised form November 10, 2010.

Address correspondence to: Axel Weber, Liebigstrasse 20a, D-04103 Leipzig, Germany. Phone: 49.0341.9726114; Fax: 49.0341.9726099; E-mail: axel.weber@medizin.uni-leipzig.de.
1. Mortuza FY, et al. Minimal residual disease tests provide an independent predictor of clinical outcome in adult acute lymphoblastic leukemia. J Clin Oncol. 2002;20(4):1094-1104.

2. Flohr T, et al. Minimal residual disease-directed risk stratification using real-time quantitative PCR analysis of immunoglobulin and T-cell receptor gene rearrangements in the internationalmulticenter trial AIEOP-BFM ALL 2000 for childhood acute lymphoblastic leukemia. Lenkemia. 2008;22(4):771-782.

3. Ignatiadis $M$, et al. Different prognostic value of cytokeratin-19 mRNA positive circulating tumor cells according to estrogen receptor and HER2 status in early-stage breast cancer. J Clin Oncol. 2007; 25(33):5194-5202.

4. Janni W, Rack B, Lindemann K, Harbeck N. Detection of micrometastatic disease in bone marrow: is it ready for prime time? Oncologist. 2005;10(7):480-492.

5. Cristofanilli $\mathrm{M}$, et al. Circulating tumor cells, disease progression, and survival in metastatic breast cancer. N Engl J Med. 2004;351(8):781-791.

6. Diehl F, et al. Circulating mutantDNA to assess tumor dynamics. Nat Med. 2008;14(9):985-990.

7. Spyridonidis A, Bernhardt W, Fetscher S, Behringer D, Mertelsmann R, Henschler R. Minimal residual disease in autologous hematopoietic harvests from breast cancer patients. Ann Oncol. 1998;9(8):821-826.

8. Santarius T, Shipley J, Brewer D, Stratton MR, Cooper CS. A census of amplified and overexpressed human cancer genes. Nat Rev Cancer. 2010;10(1):59-64.

9. Leary RJ, et al. Integrated analysis of homozygousdeletions, focal amplifications, and sequence alterations in breast andcolorectal cancers. Proc Natl Acad Sci U S A. 2008;105(42):16224-16229.

10. Parsons DW, et al. An integrated genomicanalysis of human glioblastoma multiforme. Science. 2008; 321(5897):1807-1812.

11. Schwab M, et al. Amplified DNA with limited homology to myc cellular oncogene is shared by human neuroblastoma cell lines and a neuroblastoma tumour. Nature. 1983;305(5931):245-248.

12. Jones TA, Flomen RH, Senger G, Nizetic D, Sheer $D$. The homeobox gene MEIS1 is amplified in IMR-
32 and highly expressed in other neuroblastoma cell lines. Eur J Cancer. 2000;36(18):2368-2374.

13. Spieker N, et al. The MEIS1 oncogene is highly expressed in neuroblastoma and amplified in cell line IMR32. Genomics. 2001;71(2):214-221.

14. Oberthuer A, et al. Characterization of a complex genomic alteration on chromosome 2 pthat leads to four alternatively spliced fusion transcripts in the neuroblastoma cell lines IMR-5, IMR-5/75 and IMR-32. Gene. 2005;363:41-50.

15. Weber A, Starke S, Bergmann E, Christiansen H. The coamplification pattern of the MYCN amplicon is an invariable attribute of most MYCN-amplified human neuroblastomas. Clin Cancer Res. 2006; 12(24):7316-7321.

16. Livak KJ, Schmittgen TD. Analysis of relative gene expression data using real-time quantitative PCR and the 2(-Delta Delta C(T)) method. Methods. 2001; 25(4):402-408.

17. Liu W, et al. Copy number analysis indicates monoclonal origin of lethal metastatic prostate cancer. Nat Med. 2009;15(5):559-565.

18. Wang $X$, et al. Evidence for common clonal origin of multifocal lung cancers. J Natl Cancer Inst. 2009; 101(8):560-570.

19. Bignell G, et al. Architectures of somatic genomic rearrangement in human cancer amplicons at sequencelevel resolution. Genome Res. 2007;17(9):1296-1303.

20. Szczepanski T, Orfão A, van der Velden VH, San Miguel JF, van Dongen JJ. Minimal residual disease in leukaemia patients. Lancet Oncol. 2001;2(7):409-417.

21. Borowitz MJ, et al. Minimal residual disease detection in childhood precursor-B-cell acute lymphoblastic leukemia: relation to other risk factors. Leu kemia. 2003;17(8):1566-1572.

22. Thörn I, et al. Monitoring minimal residual disease with flow cytometry, antigen-receptor gene rearrangements and fusion transcript quantification in Philadelphia-positive childhood acute lymphoblastic leukemia. Leuk Res. 2009;33(8):1047-1054.

23. Kern W, Haferlach C, Haferlach T, Schnittger S. Monitoring of minimal residual disease in acute myeloid leukemia. Cancer. 2008;112(1):4-16.
24. Lambooy LH, et al. Real-time analysis of tyrosine hydroxylase gene expression: a sensitive and semiquantitative marker for minimal residual disease detection of neuroblastoma. Clin Cancer Res. 2003; 9(2):812-819.

25. Stutterheim J, et al. PHOX2B is a novel and specific marker for minimal residual disease testing in neuroblastoma. J Clin Oncol. 2008;26(33):5443-5449.

26. Scholl C, et al. The prognostic value of MLL-AF9 detection in patients with $\mathrm{t}(9 ; 11)(\mathrm{p} 22 ; \mathrm{q} 23)$-positive acute myeloid leukemia. Haematologica. 2005; 90(12):1626-1634.

27. Schnittger S, et al. Minimal residual disease levels assessed by NPM1 mutation- pecific RQ-PCR provide important prognostic information in AML. Blood. 2009;114(11):2220-2231.

28. Hirt C, Schüler F, Dölken G. Minimal residual disease (MRD) in follicular lymphoma in the era of immunotherapy with rituximab. Semin Cancer Biol. 2003; 13(3):223-231.

29. Diehl F, et al. Detection and quantification of mutations in the plasma of patients with colorectal tumors. Proc Natl Acad Sci U S A. 2005;102(45):16368-16373.

30. Van der Velden VH, et al. Prognostic significance of minimal residual disease in infants with acute lymphoblastic leukemia treated within the Interfant99 protocol. Leukemia. 2009;23(6):1073-1079.

31. Stephens PJ, et al. Complex landscapes of somatic rearrangement in human breast cancer genomes. Nature. 2009;462(7276):1005-1010.

32. Leary RJ, et al. Development of personalized tumor biomarkers using massively parallel sequencing. Sci Transl Med. 2010;2(20):20ra14.

33. Brodeur GM, et al. Revisions of the international criteria for neuroblastoma diagnosis, staging, and response to treatment. JClin Oncol. 1993;11(8):1466-1477.

34. Berthold F, et al. The role of chemotherapy in the treatment of children with neuroblastoma stage IV: the GPO (German Pediatric Oncology Society) experience. Klin Padiatr. 1990;202(4):262-269.

35. Berthold F, Hero B. Neuroblastoma: Current drug recommendations as part of the total treatment approach. Drugs. 2000;59(6):1261-1277. 